\title{
Histomorphometric profile of the corneal response to short-term reverse-geometry orthokeratology lens wear in primate corneas: a pilot study
}

\begin{abstract}
Purpose: To investigate the histological changes in primate cornea induced by short-term overnight orthokeratology (OK).

Methods: Nine young adult primates were used. One animal served as negative control. The remaining 8 animals wore reverse-geometry OK lenses for periods of 4, 8, 16, and 24 hours on 1 eye with the other eye as control. Central and midperipheral corneal thickness, as well as ultrastructural changes in corneal epithelium, stroma and endothelium in response to $\mathrm{OK}$ lenses, were evaluated.

Results: OK significantly reduced the thickness of the central cornea in all treatment groups. The central corneal thinning was both stromal and epithelial in origin. Substantial midperipheral corneal thickening was seen in 16-hour and 24-hour lens-wear groups and this effect was both stromal and epithelial in origin as well. Histology evidence indicated the primary epithelial response in the central cornea was compression of cells that resulted in wing cells becoming shorter and basal cells being squatted rather than lost or migration of cell layers. These pronounced cell shape changes occurred without compromising the structural integrity of the desmosomes. The thickened corneal epithelium has normal cell layers. The squamous cells have larger surface sizes and are composed of oval instead of flattened nuclei. This implied delayed surface cell exfoliation at the thickened midperipheral epithelium. Physical presence of $\mathrm{OK}$ lens over the cornea did not influence the microstructures of microvilli and microplicae, endothelium, and collagen distribution.

Conclusions: The primate cornea, particularly the corneal epithelium, responds rapidly to the application of reverse-geometry OK lenses with significant epithelial cell shape alterations with short-term OK lens wear. This finding suggests that the corneal epithelium is moldable in response to the physical forces generated by the OK lenses.
\end{abstract}

Keyword: Overnight orthokeratology; Cornea; Histological changes; Primate 